\title{
AXISYMMETRIC FREE VIBRATION OF LAYERED CYLINDRICAL SHELL FILLED WITH FLUID
}

\author{
M.D. Nurul IZYAN and Nur Ain Ayunni SABRI \\ Faculty of Entrepreneurship and Business, Universiti Malaysia Kelantan, Pengkalan Chepa \\ 16100 Kota Bharu, Kelantan, MALAYSIA
}

A.K. Nor HAFIZAH

Koleg Genius Insan, Universiti Sains Islam Makaysia, Bandar Baru Nilai, 71800, Nilai, Negari Sembilan, MALAYSIA

Department of Mathematical Sciences, Faculty of Science Universiti Teknologi Malaysia 81310 Johor Bahru, Johor, MALAYSIA

\author{
D.S. SANKAR \\ School of Applied Sciences and Mathematics, Universiti Teknologi Brunei, Jalan Tungku Link \\ Gadong, BE1410, Bandar Seri Begawan, BRUNEI DARUSSALAM \\ K.K. VISWANATHAN* \\ Department of Mathematical Sciences, Faculty of Science Universiti Teknologi Malaysia \\ 81310 Johor Bahru, Johor, MALAYSIA \\ Ship \& Offshore Extreme Technology Industry-Academic Cooperation Research Center \\ Inha University, 100 Inha-Ro, Incheon, 22212, SOUTH KOREA \\ E-mails: visu20@yahoo.com, viswanathan@utm.my
}

\begin{abstract}
The aim of the study is to analyse the axisymmetric free vibration of layered cylindrical shells filled with a quiescent fluid. The fluid is assumed to be incompressible and inviscid. The equations of axisymmetric vibrations of layered cylindrical shell filled with fluid, on the longitudinal and transverse displacement components are obtained using Love's first approximation theory. The solutions of displacement functions are assumed in a separable form to obtain a system of coupled differential equations in terms of displacement functions. The displacement functions are approximated by Bickley-type splines. A generalized eigenvalue problem is obtained and solved numerically for a frequency parameter and an associated eigenvector of spline coefficients. Two layered shells with three different types of materials under clamped-clamped boundary conditions are considered. Parametric studies are made on the variation of the frequency parameter with respect to length-to-radius ratio and length-to-thickness ratio.
\end{abstract}

Key words: axisymmetric, free vibration, cylindrical shell, Love's first approximation theory, inviscid fluid, spline approximation, eigenvalues.

\section{Introduction}

Axisymmetric vibration has been applied widely to thin and laminated cylindrical shell. The laminated composite shell is used in industry such as in piping systems, pressure vessels as well as storage tanks due to high stiffness and strength to weight ratio. Different theories of shells have been used to determine the frequencies of the shell. Moreover, the presence of a liquid in a shell structure significantly affects the vibration of the shell structure in terms of frequencies.

\footnotetext{
${ }^{*}$ To whom correspondence should be addressed.
} 
There are numerous studies on the behaviour cylindrical shells under axisymmetric vibration. Forsberg [1] studied the axisymmetric and beam-type vibrations of thin cylindrical shells using Flugge's shell equations and the results were compared with exact solutions. Wang et al. [2] investigated a multiple-shell model in order to study the axisymmetric and beam vibrations. By applying simply supported end conditions, results were obtained which showed that for lower circumferential wave numbers, $n=0-10$, the lowest frequency always corresponds to the minimum half-axial wave number $m=1$. Furthermore, as the wave vector decreases, the lowest frequency decreases and the associated mode shifts from $\mathrm{R}$ mode with larger $n$ to a coaxial B mode with $n=1$. Viswanathan et al. [3] examined the axisymmetric vibrations of layered cylindrical shells of variable thickness using spline approximation. Love's first approximation theory was used to determine the frequencies of two-layered cylindrical shells with different types of boundary conditions. The axisymmetric free vibration of layered conical shells using the Chebyshev polynomial was investigated by Viswanathan et al. [4] to obtain the vibrations of the shell under clamped-clamped boundary conditions. Lopatin and Morozov [5] provided a solution of the axisymmetric vibration problem for the orthotropic cylindrical shell with rigid weightless end disks. By implementing the clamped-clamped beam functions, the shell deflection was determined, and the third derivatives of these functions were used to approximate the axial displacement. The solution was solved by using the Ritz method.

Besides that, studies on axisymmetric cylindrical shells filled with fluid, partially filled with fluid as well as submerged shell were made. Lakis and Sinno [6] studied the axisymmetric and beam-like cylindrical shells, partially filled with liquid, implemented Sanders' theory. Free vibration characteristics of anisotropic thin cylindrical shells were studied by employing Finite Element Method (FEM). Sinha et al. [7] investigated the isotropic cylindrical shell and exact solutions were obtained. Axisymmetric waves propagating along fluid-loaded cylindrical shells within the framework of linear elasticity and classical perfect-slip boundary conditions at the solid-fluid interface were investigated. Shen et al. [8] examined the beam-mode stability of fluid-conveying shell systems with clamped-free (cantilevered) boundary conditions using the Flugge shell theory. The problem was solved by using FEM.

The singular Boundary Method (SBM) was used by Vasyl et al. [9] to analyse the free vibration of compound liquid-filled shells. Results obtained with proposed SBM through numerical experiments are in good agreement with the Boundary Element Method (BEM) for axisymmetric problems of liquid vibrations. It was concluded that the singular boundary method has the essential advantage in CPU time calculations, and it does not require thorough gridding and avoids calculation of complicated singular integrals is avoided. George and Tatiana [10] examined an axisymmetric vibration of the cylindrical shell loaded with pointed masses using the finite element method. The Kirchoff-Love theory with additional inertia in the form of "mass belt" of zero width was considered in the study. Axisymmetric wave propagation in an elastic periodic cylindrical shell under internal heavy fluid loading was studied by Sorokin et al. [11]. The governing equations, which describe propagation of free axisymmetric waves in a pre-stressed isotropic homogeneous cylindrical shell with fluid loading were implemented in the study.

In this study, the problem of axisymmetric vibration of thin elastic circular cylindrical shells filled with fluid is considered. The frequencies are determined by the using spline method. The method of spline over a two-point boundary value problem with cubic splines is successfully studied by Bickley [12] . Results showed that the spline approximation used a lower order approximation, which yields a better accuracy. There are numerous studies on the vibrational behavioral of shells with and without fluid using the spline method such as the vibration of layered cylindrical shells (using spline method) [13], the vibration of antisymmetric angle-ply composite annular plates of variable thickness [14], free vibration of angle-ply laminated conical shell with linear and exponential thickness variations [15], free vibration of layered cylindrical shells filled with fluid using Love's first approximation theory [16], free vibration of antisymmetric angle-ply layered circular cylindrical shells filled with a quiescent fluid under first order shear deformation theory [17], free vibration of cross-ply layered circular cylindrical shells filled with a quiescent fluid under first order shear deformation theory [18], free vibration of symmetrically layered angle-ply cylindrical shells filled with fluid under first order shear deformation theory (FSDT) [19].

Employing Love's first approximation theory, the governing equations are coupled in the longitudinal and transverse displacement components. The layers are considered to be thin, elastic and 
specially orthotropic or isotropic and assumed to be bonded perfectly together and to move without interface slip. The fluid is assumed to be incompressible and the flow of the fluid is inviscid. By assuming the displacement components in a separable form, a system of coupled differential equations in displacement functions is obtained. Then, the displacement functions are approximated by Bickley-type splines which are cubic and quintic. Collocation with these splines yields a set of field equations together with the equations of boundary conditions. Hence, it reduces to a system of homogeneous simultaneous algebraic equations on the assumed spline coefficients which leads to a generalized eigenvalue problem. The eigenvalue problem is solved using eigensolution technique to obtain as many eigenfrequencies as required. From the eigenvectors the spline coefficients are computed from which the mode shapes are constructed. In this problem, two layers of a composite elastic shell with different types of material such as S-glass Epoxy (SGE), High Strength Graphite Epoxy (HSG) and PRD-490 I I I epoxy (PRD) are considered. The parametric study showed the effect of length-to-radius ratio and length-to-thickness ratio under clamped-clamped boundary conditions on frequency parameters.

\section{Theoretical formulation}

\subsection{Equations of the shell}

Consider a thin layered circular cylindrical shell of length $\ell$, constant thickness $h$, radius $r$. Each layer is assumed to be homogeneous, linearly elastic and isotropic or specially orthotropic. The $x$ coordinate of the shell is taken along the longitudinal direction, $\theta$ and the $z$ coordinate are in the circumferential and radial direction, respectively. Equations of motion for the cylindrical shell are written as

$$
\begin{aligned}
& \frac{\partial N_{x}}{\partial x}+\frac{1}{r} \frac{\partial N_{\theta x}}{\partial \theta}=\rho h \frac{\partial^{2} u}{\partial t^{2}} \\
& \frac{\partial^{2} M_{x}}{\partial x^{2}}+\frac{2}{r} \frac{\partial M_{\theta x}}{\partial x \partial \theta}+\frac{1}{r^{2}} \frac{\partial^{2} M_{\theta}}{\partial \theta^{2}}-\frac{N_{\theta}}{r}=\rho h\left(\frac{\partial^{2} w}{\partial t^{2}}-\frac{p}{\rho h}\right)
\end{aligned}
$$

where $N_{x}, N_{\theta}$ and $N_{x \theta}$ are the force resultants, $M_{x}, M_{\theta}$ and $M_{x \theta}$ are the moments resultants and $p$ is the pressure. They are defined as

$$
\begin{aligned}
& \left(N_{x}, N_{\theta}, N_{x \theta}\right)=\int_{z}\left(\sigma_{x}, \sigma_{\theta}, \sigma_{x \theta}\right) d z, \\
& \left(M_{x}, M_{\theta}, M_{x \theta}\right)=\int_{z}\left(\sigma_{x}, \sigma_{\theta}, \sigma_{x \theta}\right) z d z .
\end{aligned}
$$

The strain-displacement relations of the circular cylindrical shell are as follows

$$
\begin{aligned}
& \varepsilon_{x}=\frac{\partial u}{\partial x}, \quad \varepsilon_{\theta}=\frac{1}{r} \frac{\partial v}{\partial \theta}+\frac{w}{r}, \quad \gamma_{x \theta}=\frac{\partial v}{\partial x}+\frac{1}{r} \frac{\partial u}{\partial \theta} \\
& \kappa_{x}=-\frac{\partial^{2} w}{\partial x_{2}}, \quad \kappa_{\theta}=\frac{1}{r^{2}} \frac{\partial v}{\partial \theta}-\frac{1}{r^{2}} \frac{\partial^{2} w}{\partial \theta^{2}}, \quad \tau_{x \theta}=\frac{1}{r} \frac{\partial v}{\partial x}-\frac{2}{r} \frac{\partial^{2} w}{\partial x \partial \theta} .
\end{aligned}
$$

The stress-strain relations of the $k$-th layer by neglecting the transverse normal stress and strain are defined as 


$$
\left(\begin{array}{c}
\sigma_{x}^{(k)} \\
\sigma_{\theta}^{(k)} \\
\tau_{x \theta}^{(k)}
\end{array}\right)=\left(\begin{array}{ccc}
Q_{11}^{(k)} & Q_{12}^{(k)} & 0 \\
Q_{12}^{(k)} & Q_{22}^{(k)} & 0 \\
0 & 0 & Q_{66}^{(k)}
\end{array}\right)\left(\begin{array}{c}
\varepsilon_{x}^{(k)} \\
\varepsilon_{\theta}^{(k)} \\
\gamma_{x \theta}^{(k)}
\end{array}\right)
$$

Applying Eq.(2.3) into Eq.(2.4) and then substituting into Eq.(2.2), the force and moment resultants can be obtained as

$$
\left(\begin{array}{c}
N_{x} \\
N_{\theta} \\
N_{x \theta} \\
M_{x} \\
M_{\theta} \\
M_{x \theta}
\end{array}\right)=\left(\begin{array}{cccccc}
A_{11} & A_{12} & 0 & B_{11} & B_{12} & 0 \\
A_{12} & A_{22} & 0 & B_{12} & B_{22} & 0 \\
0 & 0 & A_{66} & 0 & 0 & B_{66} \\
B_{11} & B_{12} & 0 & D_{11} & D_{12} & 0 \\
B_{12} & B_{22} & 0 & D_{12} & D_{22} & 0 \\
0 & 0 & B_{66} & 0 & 0 & D_{66}
\end{array}\right)\left(\begin{array}{c}
\partial u / \partial x \\
\partial v / r \partial \theta+w / r \\
\partial v / \partial x+\partial u / r \partial \theta \\
\partial^{2} w / \partial x^{2} \\
\partial v / r^{2} \partial \theta-\partial^{2} w / r^{2} \partial \theta^{2} \\
\partial v / r \partial x-2 \partial^{2} w / r \partial x \partial \theta
\end{array}\right)
$$

where $z_{k}$ is the distance from the midsurface to the surface of the $k$-th layer. $A_{i j}, B_{i j}$ and $D_{i j}$ are the extensional rigidities, the bending-stretching coupling rigidities and the bending rigidities defined by

$$
\begin{aligned}
& A_{i j}=\sum_{k=1} Q_{i j}^{k}\left(z_{k}-z_{k-1}\right), \\
& B_{i j}=\frac{1}{2} \sum_{k=1} Q_{i j}^{k}\left(z_{k}^{2}-z_{k-1}^{2}\right), \\
& D_{i j}=\frac{1}{3} \sum_{k=1} Q_{i j}^{k}\left(z_{k}^{3}-z_{k-1}^{3}\right)
\end{aligned}
$$

with $i, j=1,2,6$.

For a thin shell, $Q_{i j}^{k}$ is the reduced stiffness defined as

$$
Q_{11}^{k}=\frac{E_{x}^{k}}{1-v_{x \theta}^{k} v_{\theta x}^{k}}, \quad Q_{12}^{k}=\frac{v_{\theta x}^{k} E_{x}^{k}}{1-v_{x \theta}^{k} v_{\theta x}^{k}}, \quad Q_{22}^{k}=\frac{E_{\theta}^{k}}{1-v_{x \theta}^{k} v_{\theta x}^{k}}, \quad Q_{66}^{k}=G_{x \theta}^{k}
$$

Using Eq.(2.5) in Eq.(2.1), the equations of equilibrium in terms of $u$ and $w$ displacements are obtained in the form

$$
\left(\begin{array}{ll}
L_{11} & L_{12} \\
L_{21} & L_{22}
\end{array}\right)\left(\begin{array}{l}
u \\
w
\end{array}\right)=\left(\begin{array}{l}
0 \\
0
\end{array}\right)
$$

The differential operators $L_{i j}(i=1,2 ; j=1,2)$ are the differential operators given as 


$$
\begin{aligned}
& L_{11}=A_{11} \frac{\partial^{2}}{\partial x^{2}}+\frac{A_{66}}{r^{2}} \frac{\partial^{2}}{\partial \theta^{2}}-\rho h \frac{\partial^{2}}{\partial t^{2}} \\
& L_{12}=\frac{A_{12}}{r} \frac{\partial}{\partial x}-B_{11} \frac{\partial^{3}}{\partial x^{3}}-\left(\frac{B_{12}}{r^{2}}+\frac{2 B_{66}}{r^{2}}\right) \frac{\partial^{2}}{\partial x \partial \theta^{2}}, \\
& L_{21}=B_{11} \frac{\partial^{3}}{\partial x^{3}}+\left(\frac{2 B_{66}}{r^{2}}+\frac{B_{12}}{r^{2}}\right) \frac{\partial^{3}}{\partial x \partial \theta^{2}}-\frac{A_{12}}{r} \frac{\partial}{\partial x}, \\
& L_{22}=\frac{2 B_{12}}{r} \frac{\partial^{2}}{\partial x^{2}}+\frac{2 B_{22}}{r^{3}} \frac{\partial^{2}}{\partial \theta^{2}}-D_{11} \frac{\partial^{4}}{\partial x^{4}}-\left(\frac{2 D_{12}}{r^{2}}+\frac{4 D_{66}}{r^{2}}\right) \frac{\partial^{4}}{\partial x^{2} \partial \theta^{2}}+ \\
& -\frac{D_{22}}{r^{4}} \frac{\partial^{4}}{\partial \theta^{4}}-\frac{A_{22}}{r^{2}}-\rho h\left(1+\frac{\rho_{f}}{\rho_{s} h} \frac{J_{n}(r)}{J_{n}^{\prime}(r)}\right) \frac{\partial^{2}}{\partial t^{2}} .
\end{aligned}
$$

The stress resultants and moment resultants are expressed in terms of longitudinal and transverse displacements; $u, w$ of the reference surface. The circumferential displacement $v$ is neglected since only the axisymmetric vibrations are studied. Therefore, the displacement components $u$ and $w$ are assumed in the form of

$$
\begin{aligned}
& u(x, t)=U(x) e^{i \omega t}, \\
& w(x, t)=W(x) e^{i \omega t}
\end{aligned}
$$

where $x$ is the longitudinal, $\omega$ is the angular frequency of vibration and $t$ is the time.

\subsection{Fluid structure interaction equations}

The fluid is assumed to be incompressible. Irrotational flow of an inviscid fluid undergoing small oscillations is expressed as wave equation. The equation of motion of the fluid can be written in the cylindrical coordinates system $(x, \theta, r)[20]$

$$
\frac{\partial^{2} p}{\partial r^{2}}+\frac{1}{r} \frac{\partial p}{\partial r}+\frac{1}{r^{2}} \frac{\partial^{2} p}{\partial \theta^{2}}+\frac{\partial^{2} p}{\partial x^{2}}=\frac{\partial^{2} p}{c^{2} \partial t^{2}}
$$

where $t$ is the time, $p$ is the pressure and $c$ is the sound of speed of the fluid. The $x$ and $\theta$-coordinates are the same as those of the shell, where the $r$-coordinate is taken from the $x$-axis of the shell.

The associated form of the pressure field in the contained fluid, which satisfies Eq. (2.8) is assumed in the form of

$$
p(r, \theta, x, t)=\psi(x) \cos (n \theta) J_{n}(r) e^{i \omega t}
$$

where $J_{n}$ is the Bessel function of order $n$ and circumferential modal parameter $n$. 
To ensure that the fluid remains in contact with the shell wall, the fluid radial displacement and shell radial displacement must be equal at the interface of the shell inner wall and the fluid. This coupling condition then

$$
-\frac{1}{i \omega \rho_{f}} \frac{\partial p}{\partial r}=\frac{\partial w}{\partial t} .
$$

Therefore,

$$
\psi(x)=\frac{\omega^{2} \rho_{f}}{J_{n}^{\prime}(r)} w(x)
$$

where $\rho_{f}$ is the density of the contained fluid and the prime on the $J_{n}$ denotes differentiation with respect to the argument $r$.

The following non-dimensional parameters are introduced

$$
\begin{aligned}
& L=\frac{\ell}{r} ; \text { length parameter, } \\
& X=\frac{x}{\ell} ; \text { distance coordinate, } \\
& R=\frac{r}{\ell} ; \text { radius parameter, } \\
& \mathrm{H}=\frac{h}{r} ; \text { thickness parameter, } \\
& \lambda=\omega \ell \sqrt{\frac{R_{0}}{A_{11}}} \text {; frequency parameter, } \\
& \delta_{k}=\frac{h_{k}}{h} ; \text { relative layer thickness of k-th layer. }
\end{aligned}
$$

In obtaining equations of shell with fluid, substituting Eq.(2.7) into Eq.(2.6) together with the Eq.(2.9) and then applying the non-dimensional parameters, we obtain the equation of the motion of the coupled system in symmetric form as

$$
\left(\begin{array}{ll}
L_{11} & L_{12} \\
L_{21} & L_{22}
\end{array}\right)\left(\begin{array}{l}
U \\
W
\end{array}\right)=\left(\begin{array}{l}
0 \\
0
\end{array}\right)
$$

where $L_{i j}(i=1,2 ; j=1,2)$ are the differential operators given as 


$$
\begin{aligned}
& L_{11}=\frac{d^{2}}{d X^{2}}-S_{10} \frac{n^{2}}{R^{2}}+\lambda^{2}, \\
& L_{12}=-S_{4} \frac{d^{3}}{d X^{3}}+\left(\left(S_{5}+2 S_{11}\right) \frac{n^{2}}{R^{2}}+\frac{S_{2}}{R}\right) \frac{d}{d X}, \\
& L_{21}=S_{4} \frac{d^{3}}{d X^{3}}-\left(\left(S_{5}+2 S_{11}\right) \frac{n^{2}}{R^{2}}+\frac{S_{2}}{R}\right) \frac{d}{d X}, \\
& L_{22}=-S_{7} \frac{d^{4}}{d X^{4}}+2\left(S_{8}+2 S_{12}\right) \frac{n^{2}}{R^{2}} \frac{d^{2}}{d X^{2}}+\frac{2 S_{5}}{R} \frac{d^{2}}{d X^{2}}+ \\
& -\left(S_{9} \frac{n^{4}}{R^{4}}+2 S_{6} \frac{n^{2}}{R^{3}}+S_{3} \frac{1}{R^{2}}\right)+\lambda^{2}\left(1+\frac{\rho_{f}}{\rho_{s} h} \frac{J_{n}(R)}{J_{n}^{\prime}(R)}\right),
\end{aligned}
$$

with

$$
\begin{aligned}
& S_{2}=\frac{A_{12}}{A_{11}}, \quad S_{3}=\frac{A_{22}}{A_{11}}, \quad S_{4}=\frac{B_{11}}{\ell A_{11}}, \quad S_{5}=\frac{B_{12}}{\ell A_{11}}, \quad S_{6}=\frac{B_{22}}{\ell A_{11}}, \\
& S_{7}=\frac{D_{11}}{\ell^{2} A_{11}}, \quad S_{8}=\frac{D_{12}}{\ell^{2} A_{11}}, \quad S_{9}=\frac{D_{22}}{\ell^{2} A_{11}}, \quad S_{10}=\frac{A_{66}}{A_{11}}, \quad S_{11}=\frac{B_{66}}{\ell A_{11}}, \\
& S_{12}=\frac{D_{66}}{\ell^{2} A_{11}}, \quad \lambda^{2}=\frac{R_{0} \omega^{2}}{A_{11}}, \quad R_{0}=\rho h .
\end{aligned}
$$

Since the second of Eq.(2.13) contains derivatives of third order in $U$, the form of Eq.(2.13) is not convenient to the solution procedure we propose to adopt. Hence, the equations are combined within themselves and a modified set of equations is derived. To modify the equations, the first of Eq.(2.13) is differentiated with respect to $X$ and used to eliminate $U^{\prime \prime \prime}(X)$ in the third equation. Hence, the modified set of equations becomes second order in $U$ and fourth order in $W$ and is given by

$$
\left(\begin{array}{ll}
L_{11} & L_{12} \\
L_{21}^{*} & L_{22}^{*}
\end{array}\right)\left(\begin{array}{l}
U \\
W
\end{array}\right)=\left(\begin{array}{l}
0 \\
0
\end{array}\right)
$$

where $L_{i j}(i=1,2 ; j=1,2)$ are the differential operators given as 


$$
\begin{aligned}
& L_{21}^{*}=\left\{S_{4} S_{10} \frac{n^{2}}{R^{2}}-\left(S_{5}+2 S_{11}\right) \frac{n^{2}}{R^{2}}-\frac{S_{2}}{R}\right\} \frac{d^{2}}{d X^{2}}-\lambda^{2} S_{4} \frac{d}{d X}, \\
& L_{22}^{*}=\left(2\left(S_{8}+2 S_{12}\right) \frac{n^{2}}{R^{2}}+\frac{2 S_{5}}{R}-S_{4}\left(S_{5}+2 S_{11}\right) \frac{n^{2}}{R^{2}}-S_{4} \frac{S_{2}}{R}\right) \frac{d^{2}}{d X^{2}}+\left(S_{4}^{2}-S_{7}\right) \frac{d^{4}}{d X^{4}}+ \\
& -S_{9} \frac{n^{4}}{R^{4}}-2 S_{6} \frac{n^{2}}{R^{3}}-S_{3} \frac{1}{R^{2}}+\lambda^{2}\left(1+\frac{\rho_{f}}{\rho_{s} h} \frac{J_{n}(R)}{J_{n}^{\prime}(R)}\right) .
\end{aligned}
$$

\section{Solution procedure}

\subsection{Bickley-type method}

The spline approximation is a lower order approximation which yields a better accuracy than a global higher order approximation. Bickley [18] successfully tested the spline collocation method over a two-point boundary value problem with cubic splines. The displacement functions $U(X)$ and $W(X)$ are approximated by cubic and quintic spline functions $U^{*}(X)$ and $W^{*}(X)$, respectively, as follows

$$
\begin{aligned}
& U^{*}(X)=\sum_{i=0}^{2} a_{i} X^{i}+\sum_{j=0}^{N-1} b_{j}\left(X-X_{j}\right)^{3} H\left(X-X_{j}\right), \\
& W^{*}(X)=\sum_{i=0}^{4} e_{i} X^{i}+\sum_{j=0}^{N-1} f_{j}\left(X-X_{j}\right)^{5} H\left(X-X_{j}\right) .
\end{aligned}
$$

Here, $H\left(X-X_{j}\right)$ is the Heaviside step function. $N$ is the number of intervals in the range of $X \in[0,1]$ which is divided. The points of division $X=X_{s}=\frac{s}{N},(s=0,1,2, \ldots N)$ are chosen as the knots of the splines as well as the collocation points. Imposing the condition that the differential equations given by Eq. (2.14) are satisfied by these splines at the knots, a set of $(2 N+2)$ homogeneous equations into $(2 N+8)$ unknown spline coefficients $a_{i}, b_{j}, c_{i}, d_{j}, e_{i}, f_{j}(i=0,1,2,3,4 ; j=0,1,2, \ldots, N-1)$ is obtained.

\subsection{Boundary conditions}

The Clamped-Clamped (C-C) boundary conditions are used to analyse the problem which is

$$
U=0, \quad W=0, \quad \frac{d W}{d X}=0 \quad \text { at } \quad X=0 \quad \text { and } \quad X=1 .
$$

By applying any of boundary conditions, we obtain six more equations on spline coefficients. Combining these six equations with the earlier $(2 N+2)$ homogeneous equations, we get $(2 N+8)$ homogeneous equations with the same number of unknowns. Thus, these equations reduce to a system which is called a generalized eigenvalue problem of the form 


$$
[M]\{q\}=\lambda^{2}[P]\{q\}
$$

where $[M]$ and $[P]$ are matrices of order $(2 N+5) \times(2 N+5),\{q\}$ is a matrix of order $(2 N+5) \times 1 . \lambda$ is the eigenparameter and eigenvector is the spline coefficient.

\section{Results and discussions}

Table 1 shows a non-dimensional frequency for an isotropic cylindrical shell filled with a fluid under the Clamped-Clamped (C-C) boundary conditions. The results were compared with Zhang et al. [1] and Iqbal et al. [2]. To verify the efficiency of the present method comparisons of the results were made. The agreement is very good.

Table 1. Comparison of frequency for isotropic cylindrical shell filled with fluid under Clamped-Clamped (C-C) boundary conditions $(L=20, R=1, h=0.01)$.

\begin{tabular}{|c|c|c|c|c|}
\hline \multicolumn{2}{|c|}{ Mode } & \multicolumn{3}{c|}{ Frequency (Hz) } \\
\hline $\begin{array}{c}\text { Circumferential } \\
\text { Node } \mathrm{n}\end{array}$ & Axial Mode $\mathrm{m}$ & Zhang et al. [1] & Iqbal et al. [2] & Present Results \\
\hline 2 & 1 & 4.93 & 4.9083 & 5.45016 \\
\hline 2 & 2 & 11.48 & 11.3512 & 12.9516 \\
\hline 4 & 1 & 18.26 & 18.23588 & 18.0586 \\
\hline 4 & 2 & 18.73 & 18.6695 & 18.8535 \\
\hline 4 & 3 & 19.96 & 19.8425 & 21.0737 \\
\hline
\end{tabular}

To determine the behavior of the shells, an investigation on two layered cylindrical shells filled with the fluid under Clamped-Clamped (C-C) boundary conditions was conducted. Three different types of materials considered are High Strength Graphite epoxy (HSG), S-Glass Epoxy (SGE) and PRD-490 III epoxy (PRD). The variation of frequency parameters with respect to the thickness of cylinder $(H)$ and length of cylinder $(L)$ was studied.
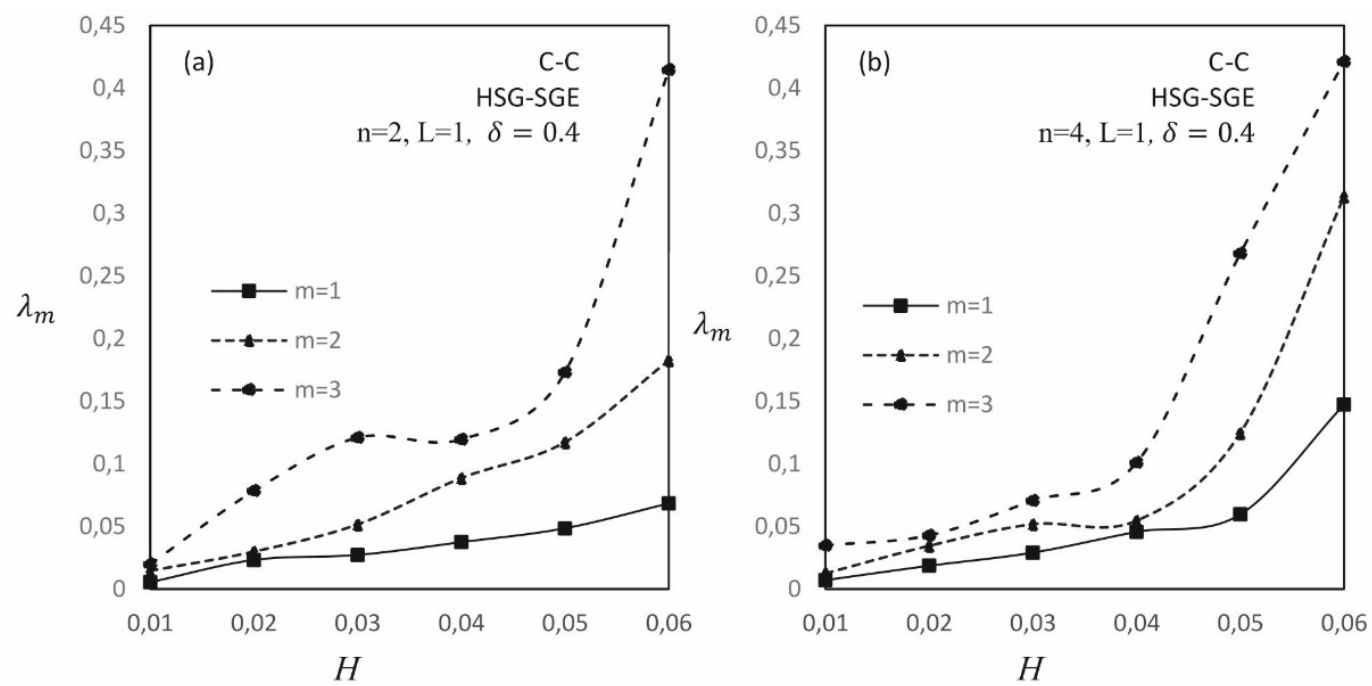

Fig.1. Effect of the thickness parameter on the frequency parameter for C-C boundary conditions (HSG-

SGE materials). 


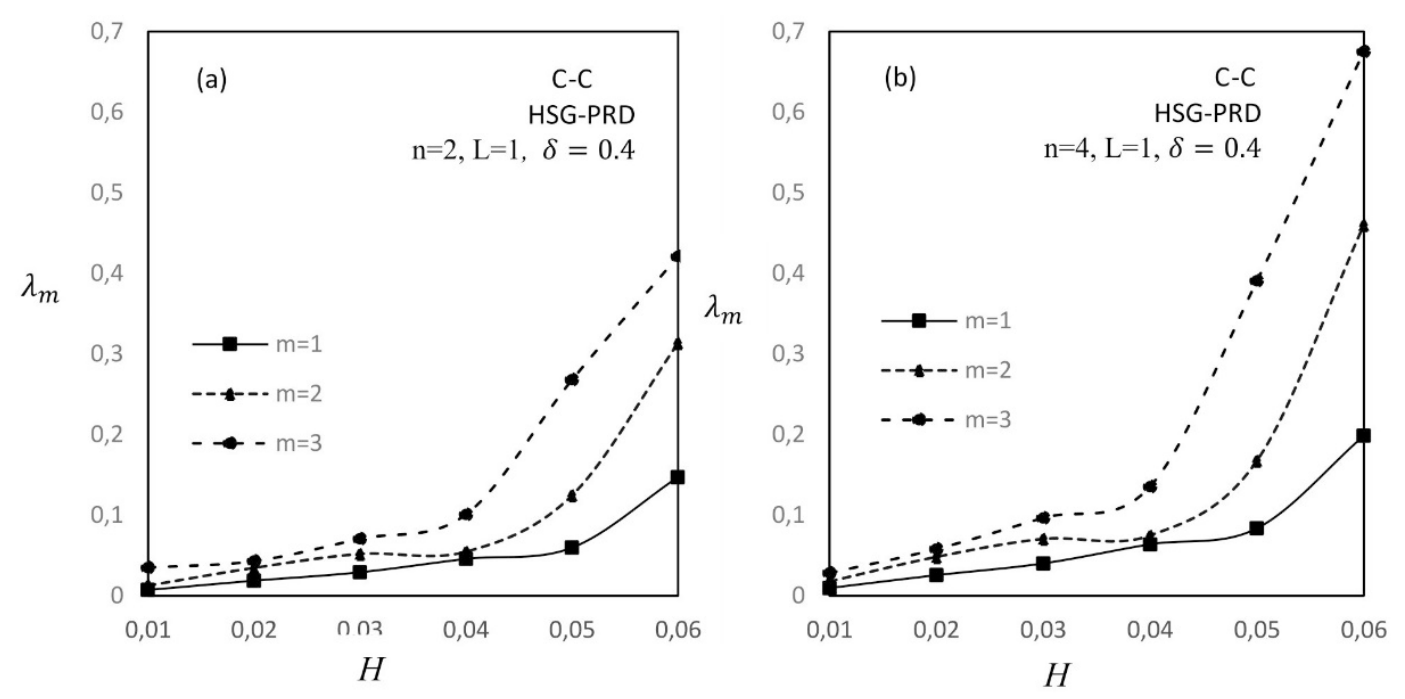

Fig.2. Effect of the thickness parameter on the frequency parameter for C-C boundary condition (HSGPRD materials).

Figure 1 shows the variation of the frequency parameter $\lambda_{m}(m=1,2,3)$ in the thickness parameter $H$ for two layers HSG-SGE materials under C-C boundary conditions. As for Fig.1(a), the values of the circumferential node number $n=2$, length $L=1$ and relative thickness layer $\delta=0.4$ are set. Meanwhile, the values of $n=4, L=1$ and $\delta=0.4$ are fixed as depicted in Fig.1(b). Generally, $\lambda_{m}(m=1,2,3)$ increases as $H$ increases. Moreover, the frequency parameter $\lambda_{m}(m=1,2,3)$ increases rapidly between $H=0.05$ and $H=0.06$. In conclusion, the values of $\lambda_{m}(m=1,2,3)$ are higher for higher values of $H$.

Figure 2 depicts how the thickness of the shell, $H$ affects the frequency parameter $\lambda_{m}(m=1,2,3)$ for two layers of composite materials with arrangement HSG-PRD under C-C boundary conditions. The effect of the frequency parameter is observed by setting the values of the length parameter $L=1$ and relative layer thickness $\delta=0.4$. Meanwhile, $n=2$ and $n=4$ are set for Fig.2(a) and Fig.2(b), respectively. The pattern of the frequencies is similar as in Fig. 1 in which when the values of $H$ increase, the frequency parameter $\lambda_{m}(m=1,2,3)$ increases. It can also be seen that in Fig.2(a), $\lambda_{m}(1,2,3)$ increases rapidly in the range $0.05<H<0.06$.

Figure 3 demonstrates the variation of the angular frequency $\omega_{m}(m=1,2,3)$ of cylindrical shells with respect to the length parameter having two layers which are HSG-SGE materials under C-C boundary conditions by fixing the values of $H=0.02$. Since the frequency parameter that $\lambda_{m}$ is explicitly a function of the length of the cylinder, hence when studying the influence of the length of the cylinder on its vibrational behaviour, the angular frequency $\omega_{m}(\mathrm{~m}=1,2,3)$ is considered instead of $\lambda_{m}$. In general, the values of $\omega_{m}(\mathrm{~m}=1,2,3)$ decrease as the length of the shell increases.

A comparison of the values $\omega_{m}(\mathrm{~m}=1,2,3)$ is made between Fig.3(a) and Fig.3(b). Results show that when $n$ changes from $n=2$ to $n=4$, the values of $\omega_{m}(\mathrm{~m}=1,2,3)$ in Fig. $3 \mathrm{~b}$ are higher than the values of $\omega_{m}(\mathrm{~m}=1,2,3)$ in Fig.3(a). From Fig.3(c) and Fig.3(d), it can also be seen that the values of $\omega_{m}(\mathrm{~m}=1,2,3)$ in Fig.3(d) are higher compared to Fig.3(c). 

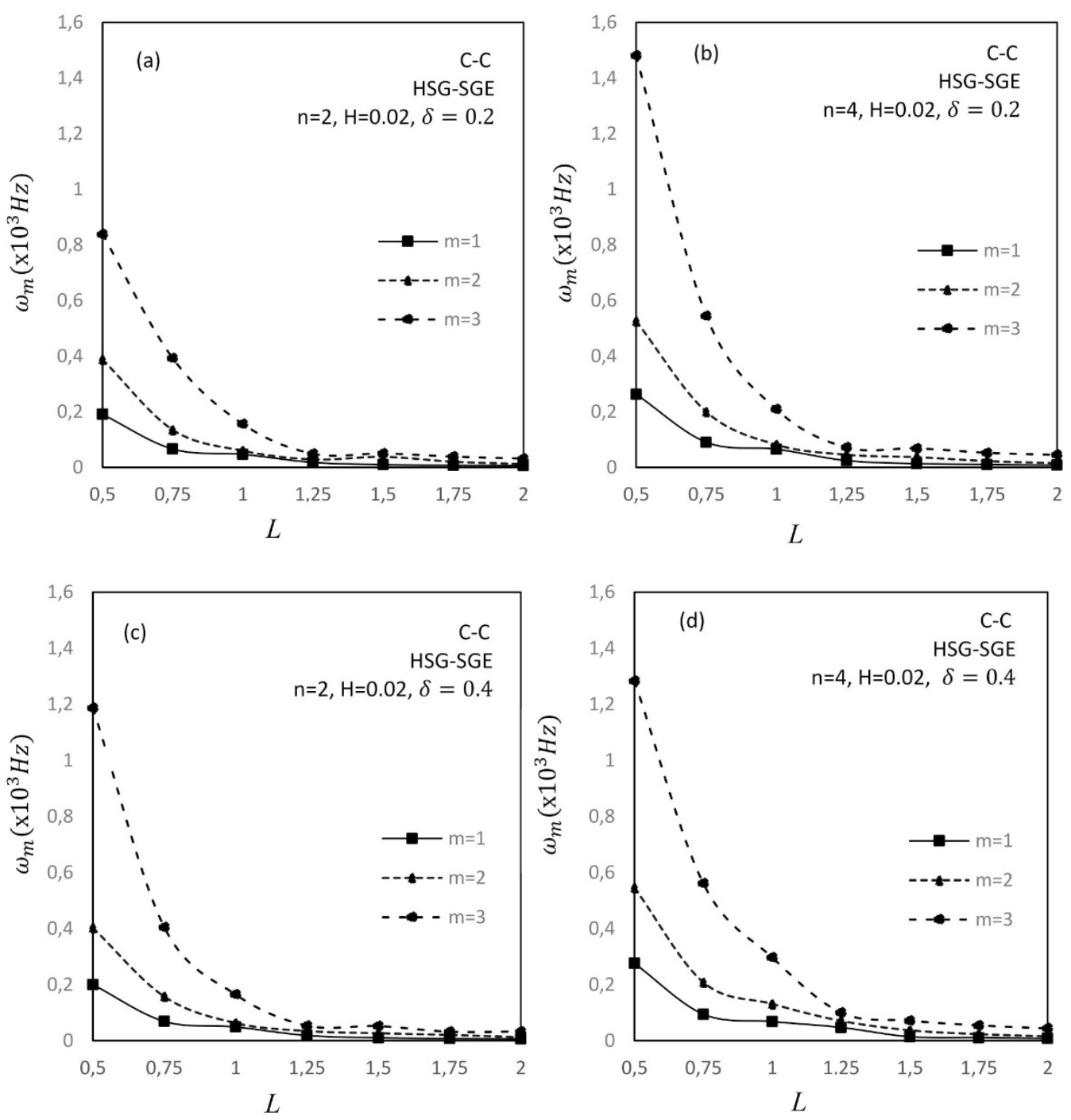

Fig.3. Effect of the length on the angular frequency for C-C boundary conditions (HSG-SGE materials).
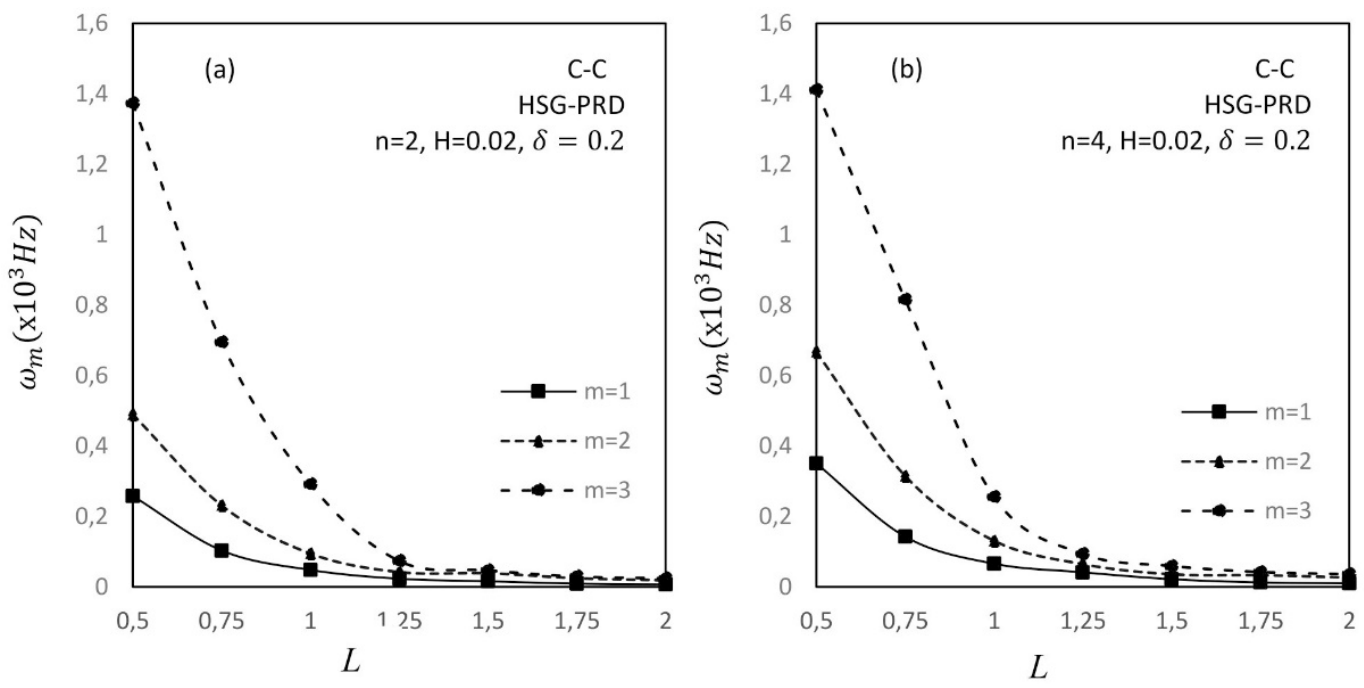

Fig.4. Effect of the length on the angular frequency for C-C boundary conditions (HSG-PRD materials). 

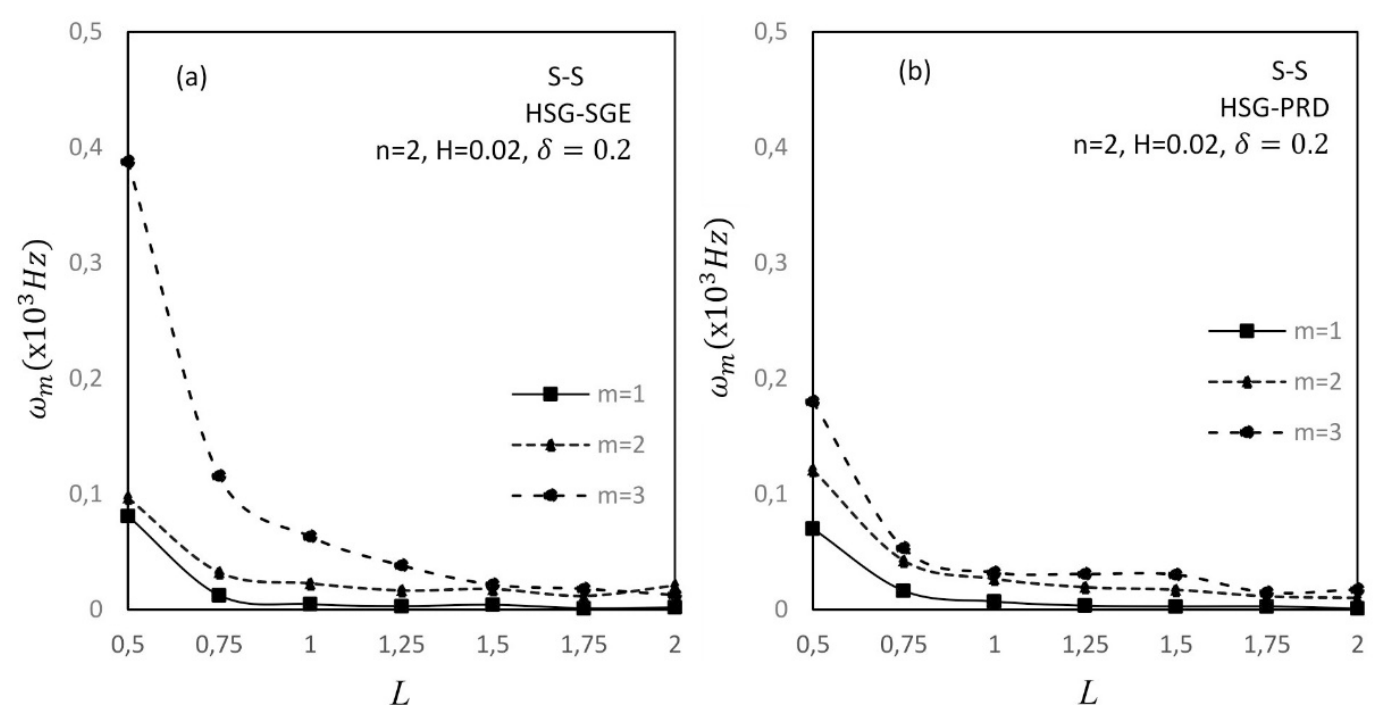

Fig.5. Effect of the length on the angular frequency for S-S boundary conditions: (a) HSG-SGE materials, (b) HSG-PRD materials).

Figure 4 depicts the variation of the angular frequency $\omega_{m}(\mathrm{~m}=1,2,3)$ with respect to the length parameter. HSG-PRD materials were considered under C-C boundary conditions with the circumferential node number $n=2$, thickness $H=0.02$ and $\delta=0.2 ; n=2$ and $n=4$ for Fig.4(a) and Fig.4(b) are fixed, respectively. It has been mentioned (Fig.3.) that the frequency $\omega_{m}(\mathrm{~m}=1,2,3)$ decreases as the length of the shell increases. The pattern of the frequency $\omega_{m}(\mathrm{~m}=1,2,3)$ in Fig.4 is similar to Fig.3.

Figure 5 illustrates to the effect of the length parameter $L$ on the angular frequency $\omega_{m}\left(\times 10^{3} \mathrm{~Hz}\right)$ of cylindrical shells having combinations of two layers which are HSG, SGE and PRD materials under C-C boundary conditions. The circumferential node number $n=2$ relative layer thickness $\delta=0.4$ and thickness $H=0.02$ are fixed. HSG-SGE materials were used in Fig.5(a) whereas HSG-PRD materials were used in Fig.5(b). From Fig.5., it is seen that as $L$ increases $\omega_{m}$ decreases for $m=1,2,3$. However, it can be observed from the figure that the decrease is fast in the range of $0<L<0.75$, meanwhile $\omega_{m}(\mathrm{~m}=1,2,3)$ decreases slowly between $0.75<L<2$.

\section{Conclusion}

The axisymmetric vibration of a two-layered cylindrical shell filled with a fluid using spline approximation was studied. The equations of the shell were considered according to Love's shell theory, and the shells were clamped at both ends. As for the fluid, the fluid is assumed to be incompressible and inviscid. The Bickley-type spline was applied to the problem to approximate the displacements. Then, the eigenvalue problem was solved to determine the frequency parameters. The impact of the length to thickness ratio and length to radius ratio on the frequency parameters was determined. It is concluded that the effects of the thickness $H$ on the frequency parameter $\lambda_{m}$ is that $\lambda_{m}$ increases as $H$ increases. The frequency parameter value increases as $L$ increases.

\section{Nomenclature}

$M_{x}, M_{\theta}, M_{x \theta} \quad-$ moment resultants in the respective directions of the shell 


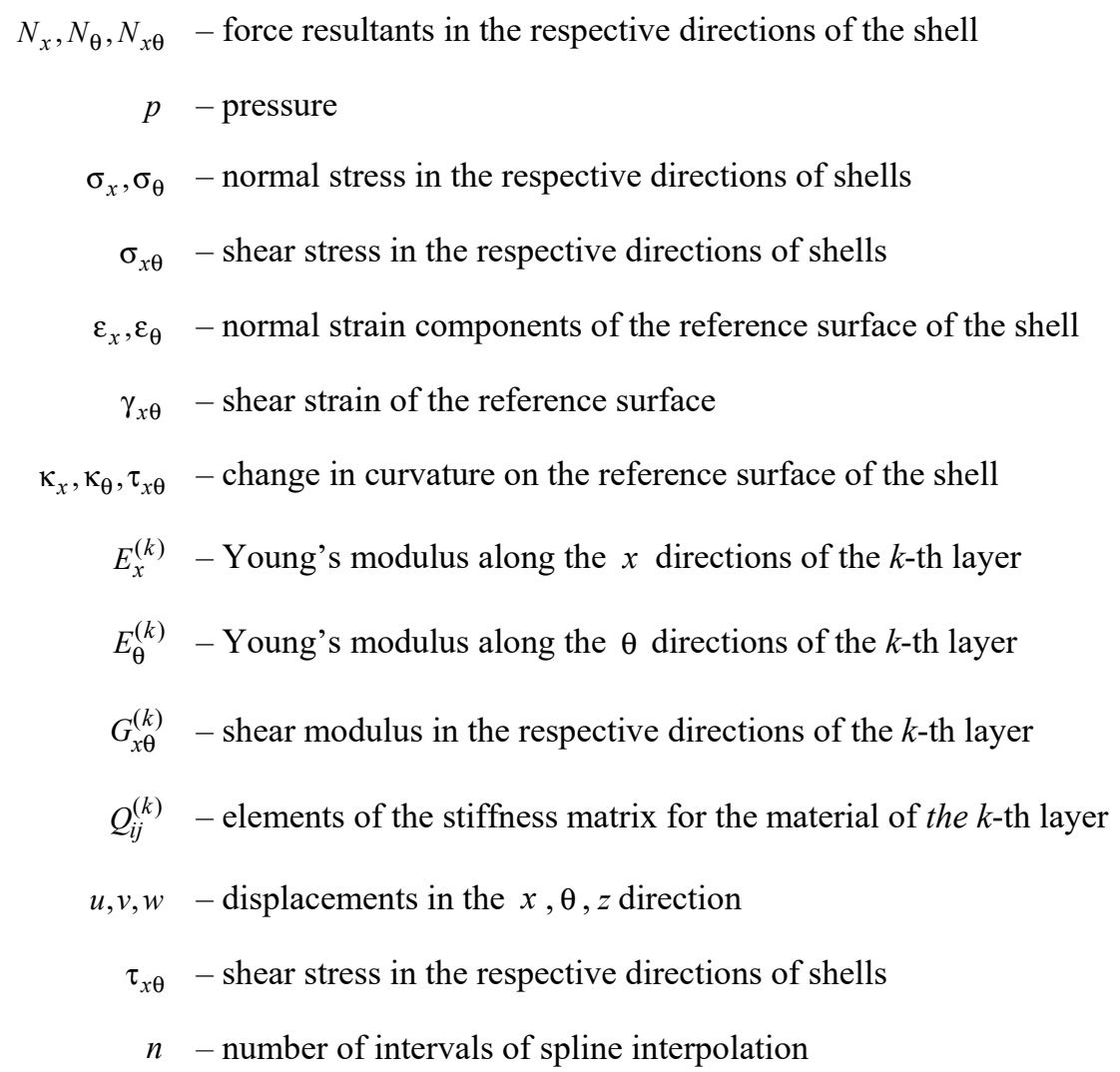

\section{References}

[1] Forsberg K. (1969): Axisymmetric and beam-type vibrations of thin cylindrical shells.- AIAA Journal, vol.7, no.2, pp.221-227.

[2] Wang C., Ru C. and Mioduchowski A. (2015): Axisymmetric and beamlike vibrations of multiwall carbon nanotubes.- Physical Review B, vol.7, No.2, pp.221-227.

[3] Viswanathan K.K., Kim K.S., Lee J.H., Lee C.H. and Lee J.B. (2008): Axisymmetric vibrations of layered cylindrical shells of variable thickness using spline function approximation.- Structural Engineering and Mechanics, vol.28, No.6, pp.749-765.

[4] Viswanathan K.K., Aziz Z. A., Lee J.H. and Nurul Izyan M.D. (2015): Axisymmetric free vibration of layered conical shells using Chebyshev polynomial with collocation method.- $16^{\text {th }}$ Asia Pacific Vibration Conference 2015 (APVC 2015), 24-26 November 2015, Manuscript ID: 63539.

[5] Lopatin A.V. and Morozov E.V. (2019): Axisymmetric vibrations of the composite orthotropic cylindrical shell with rigid weightless end disks.- Thin-Walled Structures, vol.135, pp.463-471.

[6] Lakis A.A. and Sinno M. (1992): Free vibration of axisymmetric and beam-like cylindrical shells, partially filled with liquid.- International Journal for Numerical Methods in Engineering, vol.33, No.2, pp.235-268.

[7] Sinha B.K., Plona T.J., Kostek S. and Chang S. (1992): Axisymmetric wave propagation in fluid-loaded cylindrical shells. I: Theory.- The Journal of the Acoustical Society of America, vol.92, No.2, pp.1132-1143.

[8] Shen H., Paidoussis M.P., Wen J., Yu D. and Wen X. (2014): The beam-mode stability of periodic functionallygraded-material shells conveying fluid.- Journal of Sound and Vibration, vol.333, No.10, pp.2735-2749.

[9] Vasyl V.V., Degtyariov K.G. Naumenko V.V. and Strelnikova E.A. (2019): Singular boundary method in a free vibration analysis of compound liquid-filled shells.- WIT Trans. Eng. Sci, vol.126, pp.189-200.

[10] Filippenko G.V. and Zinovieve T.V. (2020): Advances in Mechanical Engineering.- In Modern Engineering: Science and Education, Springer, Saint Petersburg, Rusia, June 2020, pp.80-91. 
[11] Sorokin S.V., Gautier F. and Pelat A. (2020): A hierarchy of models of axisymmetric wave propagation in a fluidfilled periodic cylindrical shell composed of high-contrast cells.- Mechanical Systems and Signal Processing, vol.136, pp.106487-106501.

[12] Bickley W.G. (1968): Piecewise cubic interpolation and two-point boundary problems.- Computer Journal, vol.11, pp.206-208.

[13] Viswanathanan K.K. and Navaneethakrishnan P.V. (2003): Free vibration study of layered cylindrical shells by collocation with splines.- Journal of Sound and Vibration, vol.260, No.5, pp.807-827.

[14] Hafizah A.K., Viswanathan K.K., Aziz Z.A. and Lee J.H. (2018): Vibration of antisymmetric angle-ply composite annular plates of variable thickness.- Journal of Mechanical Science and Technology, vol.32, No.5, pp.2155-2162.

[15] Viswanathan K.K., Nor Hafizah A.K. and Aziz Z.A. (2018): Free vibration of angle-ply laminated conical shell frusta with linear and exponential thickness variations.- International of Acoustics and Vibration, vol.23, No.2, pp.264-276.

[16] Nurul Izyan M.D., Viswanathan K.K., Aziz Z.A. and Prabakar K. (2016): Free vibration of layered cylindrical shells filled with fluid.- Applied Mathematics and Mechanics (English Edition), vol.37, No.6, pp.803-820.

[17] Nurul Izyan M.D., Aziz Z.A. and Viswanathan K.K. (2018): Free vibration of anti-symmetric angle-ply layered circular cylindrical shells filled with quiescent fluid under first order shear deformation theory.- Composite Structures, vol.193, pp.189-197.

[18] Nurul Izyan M.D., Aziz Z.A., Rabih Ghostine, Lee J.H. and Viswanathan K.K. (2019): Free vibration of cross-ply layered circular cylindrical shells filled with quiescent fluid under first order shear deformation theory.International Journal of Pressure Vessels and Piping, vol.170, pp.73-81.

[19] Nurul Izyan M.D. and Viswanathan K.K. (2019): Vibration of symmetrically layered angle-ply cylindrical shells filled with fluid.- PLOS ONE, vol.14, No.17, pp.1-18.

[20] Zhang X., Liu G. and Lam K. (2001): Coupled vibration analysis of fluid filled cylindrical shells using the wave propagation approach.- Applied Acoustics, vol.62, pp.229-243.

[21] Iqbal Z., Naeem M.N., Sultana N., Arshad S.H. and Shah A.G. (2009): Vibration characteristics of FGM circular cylindrical shells filled with fluid using wave propagation approach.- Applied Mathematics and Mechanics (English Edition), vol.30, No.11, pp.1393-1404.

Received: June 14, 2021

Revised: September 2, 2021 